gr-qc/9506052

MIT-CTP-2446

TUTP-95-1

Dec. 11,1995

\title{
AVERAGED ENERGY CONDITIONS AND EVAPORATING BLACK HOLES
}

\author{
L.H. Ford \\ Center for Theoretical Physics, Laboratory for Nuclear Science \\ Massachusetts Institute of Technology, Cambridge, Massachusetts 02139 \\ and \\ Institute of Cosmology, Department of Physics and Astronomy \\ Tufts University, Medford, Massachusetts 02155 \\ and \\ Thomas A. Roman \\ Institute of Cosmology, Department of Physics and Astronomy \\ Tufts University, Medford, Massachusetts 02155 \\ and \\ Department of Physics and Earth Sciences \\ Central Connecticut State University, New Britain, CT 06050"
}

*Permanent address; email: lford@pearl.tufts.edu

†Permanent address; email: roman@ccsu.ctstateu.edu 


\begin{abstract}
In this paper the averaged weak (AWEC) and averaged null (ANEC) energy conditions, together with uncertainty principle-type restrictions on negative energy ("quantum inequalities"), are examined in the context of evaporating black hole backgrounds in both two and four dimensions. In particular, integrals over only half-geodesics are studied. We determine the regions of the spacetime in which the averaged energy conditions are violated. In all cases where these conditions fail, there appear to be quantum inequalities which bound the magnitude and extent of the negative energy, and hence the degree of the violation. The possible relevance of these results for the validity of singularity theorems in evaporating black hole spacetimes is discussed.
\end{abstract}




\section{Introduction}

It is by now well-known that quantum field theory permits violations of all of the local energy conditions used in classical general relativity. These conditions are employed in a variety of ways, such as in the proofs of theorems on the occurrence of singularities in gravitational collapse and cosmology, or of cosmic censorship. Two of the weakest such local conditions are the "weak energy condition" and the "null energy condition" which state that:

$$
T_{\mu \nu} u^{\mu} u^{\nu} \geq 0
$$

for all timelike vectors $u^{\mu}$, and

$$
T_{\mu \nu} K^{\mu} K^{\nu} \geq 0
$$

for all null vectors $K^{\mu}$, respectively [1]. Note that the null energy condition follows by continuity if the weak energy condition holds. These two local conditions are satisfied by known forms of classical matter, but there are a variety of states of quantum fields which violate them [2], the most well-known of which is arguably the Casimir vacuum [3].

The extent to which quantum field theory allows violations of the local energy conditions is not yet completely clear, although progress has been made in recent years to answer this question. Two principal approaches for determining the degree of violation have been "averaged energy conditions" and "quantum inequalities." The first, originally due to Tipler [4], involves a suitable averaging of the local conditions over timelike or null geodesics. It can be shown that many of the standard results of classical general relativity obtained via global techniques can be proved using only the averaged, rather than the local, energy conditions [4]-[10]. Here we take the "averaged weak energy condition" (AWEC) to be

$$
\int_{-\infty}^{\infty} T_{\mu \nu} u^{\mu} u^{\nu} d \tau \geq 0
$$

where the integral is taken over a timelike geodesic with tangent vector $u^{\mu}$ and parameterized by the proper time $\tau$. Similarly, we take the "averaged null energy condition" (ANEC) to be

$$
\int_{-\infty}^{\infty} T_{\mu \nu} K^{\mu} K^{\nu} d \lambda \geq 0
$$

where the integral is taken over an affinely parameterized null geodesic with tangent vector $K^{\mu}$ and affine parameter $\lambda$ [11]. There has been a great deal of effort in the last several years to determine whether quantum field theory enforces averaged energy conditions. Most of this activity has been concentrated primarily on ANEC [12]-[17]. This is in part due to the discovery that violations of ANEC are required to maintain traversable wormholes [18, 19. It appears that although ANEC holds for a wide class of quantum states in a variety of spacetimes, it does not hold in an arbitrary four-dimensional curved spacetime (see Refs. [14], [15], and [16] for more 
detailed discussions). However, it is quite plausible that a suitable generalization of ANEC may hold in more general spacetimes [17, 20].

The second approach involves uncertainty principle-type inequalities, derived from quantum field theory, which restrict the magnitude and duration of negative energy fluxes or densities [20, 21, 22]. For example, one such "quantum inequality" (QI) for negative energy fluxes seen by inertial observers in two-dimensional flat spacetime has the form:

$$
|F|(\Delta \tau)^{2} \lesssim 1
$$

where $|F|$ is the magnitude of the negative energy flux and $\Delta \tau$ is its duration. This inequality implies that $\Delta E$, the amount of negative energy transmitted in time $\Delta \tau$, is limited by

$$
|\Delta E| \Delta \tau \lesssim 1
$$

Therefore, $\Delta E$ is less than the quantum uncertainty in the energy, $(\Delta \tau)^{-1}$, on the timescale $\Delta \tau$. Recently, more precise versions of these inequalities have been derived [22]. These involve an integral of the energy flux multiplied by a "sampling function", i.e., a peaked function of time with a time integral of unity and characteristic width $\tau_{0}$. A convenient choice [23] of such a function is $\tau_{0} /\left[\pi\left(\tau^{2}+\tau_{0}^{2}\right)\right]$. If the integrated flux, $\hat{F}$, is defined by

$$
\hat{F} \equiv \frac{\tau_{0}}{\pi} \int_{-\infty}^{\infty} \frac{F(\tau)}{\tau^{2}+\tau_{0}^{2}} d \tau
$$

then these inequalities may be written as

$$
\hat{F} \gtrsim-\frac{1}{16 \pi \tau_{0}^{2}}
$$

and

$$
\hat{F} \gtrsim-\frac{3}{32 \pi^{2} \tau_{0}^{4}}
$$

for all $\tau_{0}$, in two- and four-dimensions, respectively. These inequalities have the form required to prevent macroscopic violations of the second law of thermodynamics [21, 22]. It was also discovered that similar inequalities hold for a quantized massless, minimally-coupled scalar field propagating on two- and four-dimensional extreme Reissner-Nordström black hole backgrounds. These inequalities were shown to foil attempts to produce an unambiguous violation of cosmic censorship by injecting a negative energy flux into an extreme charged black hole [24, 25]. The latter results provide perhaps one of the strongest reasons for the belief that the production of large-scale effects via manipulation of negative energy may be forbidden by quantum field theory. Classically, any amount of negative energy, no matter how small, injected into an extreme black hole is enough to produce a naked singularity. Therefore, if one had any chance of producing gross effects with negative energy, the scenario discussed above would seem to have offered the best possibility of success. It should also be emphasized that the energy-time uncertainty principle was not used as input 
in the derivation of any of the QI restrictions. They arise directly from quantum field theory.

In a recent paper, we have shown that there exist deep connections between averaged energy conditions and quantum inequalities, at least in flat spacetime [20]. For a quantized massless, minimally-coupled scalar field in two- and four-dimensional Minkowski spacetime, we have derived analogous QI's to Eqs. (8) and (9) for energy density. In particular, for timelike geodesics in two-dimensional flat spacetime,

$$
\frac{\tau_{0}}{\pi} \int_{-\infty}^{\infty} \frac{\left\langle T_{\mu \nu} u^{\mu} u^{\nu}\right\rangle d \tau}{\tau^{2}+\tau_{0}^{2}} \geq-\frac{1}{8 \pi \tau_{0}^{2}}
$$

for all $\tau_{0}$. Here $\left\langle T_{\mu \nu} u^{\mu} u^{\nu}\right\rangle$ is the renormalized expectation value taken in an arbitrary quantum state $|\psi\rangle$. In the limit as $\tau_{0} \rightarrow \infty$, we "sample" the entire geodesic, and our inequality Eq. (10) reduces to AWEC. Note that it is possible that the integral in Eq. (10) converges, even though the associated AWEC integral in Eq. (3) diverges. Analogous results hold in this case for null geodesics.

For a quantized massless scalar field in a two-dimensional Minkowski spacetime with compactified spatial dimension, it was also discovered that the difference between the expectation values of $T_{\mu \nu} u^{\mu} u^{\nu}$, or $T_{\mu \nu} K^{\mu} K^{\nu}$, in an arbitrary quantum state and in the Casimir vacuum state obey AWEC and ANEC-type inequalities [20]. This is surprising since it is known [12] that AWEC and ANEC are violated for $\left\langle T_{\mu \nu} u^{\mu} u^{\nu}\right\rangle$ and $\left\langle T_{\mu \nu} K^{\mu} K^{\nu}\right\rangle$, respectively, in the (renormalized) Casimir vacuum state by itself. Such "difference inequalities" might provide new measures of the degree of energy condition violation in cases where the usual averaged energy conditions fail. This approach has recently been generalized to arbitrary two-dimensional curved spacetimes by Yurtsever [16].

For a quantized massless, minimally-coupled scalar field in four-dimensional Minkowski spacetime, we also derived the following inequality for timelike geodesics

$$
\hat{\rho}=\frac{\tau_{0}}{\pi} \int_{-\infty}^{\infty} \frac{\left\langle T_{\mu \nu} u^{\mu} u^{\nu}\right\rangle d \tau}{\tau^{2}+\tau_{0}^{2}} \geq-\frac{3}{32 \pi^{2} \tau_{0}^{4}}
$$

for all $\tau_{0}$. From this inequality, it was shown that one can derive AWEC and ANEC [20]. (The application of QI bounds to constrain the dimensions of traversable wormholes is discussed in a separate manuscript 26.)

In almost all studies of ANEC for quantum fields, the bounds on the ANEC integral have been taken from $-\infty$ to $+\infty$ [12, 14, 27]. However, a question of some interest is whether ANEC is satisfied in the spacetime of an object collapsing to form a black hole. The answer to this question might determine, for example, whether Penrose's singularity theorem [28] will still hold in the presence of local violations of the energy conditions, such as the Hawking evaporation process [29, 30]. In this case, one is usually concerned with the focusing of null geodesics which generate the boundary of the future of a trapped surface. To prove Penrose's theorem, one would want ANEC to hold over these half-complete geodesics [8, 9]. This is a stronger 
condition to impose than to demand that ANEC hold over full (i.e., past and futurecomplete) geodesics. However, even if this version of ANEC fails in some regions of a given spacetime, it may hold in enough other regions so that the conclusions of Penrose's theorem will still be valid. In addition, it should be noted that ANEC is a sufficient condition to insure continued focusing of null geodesics. One can also guarantee focusing with other conditions, for example by allowing the right-hand-side of the ANEC integral to be only periodically non-negative [7], or even negative but bounded (see Lemma 3 of Ref. [6], Eq. (5) of Ref. [9], and Ref. [17]).

In the current paper, we examine quantized massless scalar, and electromagnetic fields in the Unruh vacuum state in two- and four-dimensional Schwarzschild black hole backgrounds. We evaluate AWEC and ANEC integrals along half-complete timelike and null geodesics, respectively. Our goal is to determine the regions of these spacetimes in which the averaged energy conditions fail, and whether there exist any bounds on the extent of the violation. The paper is organized as follows. The analysis of 2D evaporating black holes is presented in Sec. (2); the results for 4D black holes are given in Sec. (3). The latter section also includes a discussion of ANEC along orbiting null geodesics. Concluding remarks are given in Sec. (4). Our units are taken to be: $\hbar=G=c=1$.

\section{2D Evaporating Black Holes}

In this section, we study AWEC and ANEC for a quantized, massless scalar field in the Unruh and Boulware vacuum states, along geodesic segments in a 2D Schwarzschild spacetime. The discussion here is a natural extension of the analysis in Ref. [31].

\subsection{Timelike Observers}

Let $u^{\mu}$ be the two-velocity of an inertial observer. The energy density in this observer's frame is given by

$$
U=T_{\mu \nu} u^{\mu} u^{\nu}
$$

In the following discussion, we understand $T_{\mu \nu}$ to denote a quantum expectation value in a specified vacuum state. The metric is

$$
d s^{2}=-C d t^{2}+C^{-1} d r^{2}
$$

where $C=1-2 M / r$. An outgoing geodesic observer's two-velocity is

$$
u^{\mu}=\left(u^{t}, u^{r}\right)=\left(\frac{d t}{d \tau}, \frac{d r}{d \tau}\right)=\left(\frac{k}{C}, \sqrt{k^{2}-C}\right) .
$$

The constant $k$ is the energy per unit rest mass. In our two-dimensional discussion, we will consider observers moving in both the Unruh and Boulware vacua. The stress tensor components in the Unruh vacuum are 32]:

$$
T_{t t}=\frac{1}{24 \pi}\left(\frac{7 M^{2}}{r^{4}}-\frac{4 M}{r^{3}}+\frac{1}{32 M^{2}}\right),
$$




$$
T_{t r}=-\frac{1}{24 \pi}\left(1-\frac{2 M}{r}\right)^{-1} \frac{1}{32 M^{2}}
$$

and

$$
T_{r r}=-\frac{1}{24 \pi}\left(1-\frac{2 M}{r}\right)^{-2}\left(\frac{M^{2}}{r^{4}}-\frac{1}{32 M^{2}}\right) .
$$

The corresponding components in the Boulware vacuum are given by:

$$
\begin{gathered}
T_{t t}=\frac{1}{24 \pi}\left(\frac{7 M^{2}}{r^{4}}-\frac{4 M}{r^{3}}\right), \\
T_{t r}=0
\end{gathered}
$$

and

$$
T_{r r}=-\frac{1}{24 \pi} \frac{M^{2}}{r^{4}}\left(1-\frac{2 M}{r}\right)^{-2} .
$$

For an outgoing timelike observer in the Unruh vacuum,

$$
T_{\mu \nu} u^{\mu} u^{\nu}=\frac{1}{24 \pi} C^{-2}\left\{k^{2}\left[\frac{6 M^{2}}{r^{4}}-\frac{4 M}{r^{3}}+\frac{1}{16 M^{2}}\right]+\frac{C M^{2}}{r^{4}}-\frac{C}{32 M^{2}}-\frac{k \sqrt{k^{2}-C}}{16 M^{2}}\right\} .
$$

Let us consider, for simplicity, the case of an observer who starts out initially very close to the horizon and is shot outward at large velocity, i.e., we are interested in the limits

$$
\epsilon \ll M, \quad k \gg 1,
$$

where $r=2 M+\epsilon$ is the observer's initial position. In this limit $k \gg C$, so from the geodesic equation, Eq. (14), we have that

$$
\tau \sim \frac{r-2 M-\epsilon}{k}+O\left(k^{-3}\right)
$$

We now wish to multiply $T_{\mu \nu} u^{\mu} u^{\nu}$ by a sampling function, i.e., a peaked function of time whose time integral is unity. In Refs. [20] and [22], this function was chosen to be

$$
\frac{\tau_{0}}{\pi} \int_{-\infty}^{\infty} \frac{d \tau}{\tau^{2}+\tau_{0}^{2}}=1
$$

where the integral was taken over complete geodesics. However, unlike in Ref. [20], here we are integrating over half-infinite geodesics. Since the function given by Eq. (24) is symmetric about $\tau=0$, in our case we may choose

$$
\frac{2 \tau_{0}}{\pi} \int_{0}^{\infty} \frac{d \tau}{\tau^{2}+\tau_{0}^{2}}=1
$$

where the proper time $\tau$ is initialized when the observer starts at $r=2 M+\epsilon$. We multiply $T_{\mu \nu} u^{\mu} u^{\nu}$ by this sampling function, use Eq. (23), and expand the numerator 
in inverse powers of $k$. The integral is performed using MACSYMA and the result is expanded in powers of $\epsilon$, yielding

$$
\begin{aligned}
\frac{2 \tau_{0}}{\pi} \int_{0}^{\infty} \frac{T_{\mu \nu} u^{\mu} u^{\nu} d \tau}{\tau^{2}+\tau_{0}^{2}} & \sim-\frac{k}{24 \pi^{2} \tau_{0}}\left[\frac{1}{\epsilon}+\frac{1}{M} \ln \left(\frac{\epsilon}{2 M}\right)+O\left(\epsilon^{0}\right)\right] \\
& +\frac{M}{3 \pi^{2} \tau_{0}^{3} k} \ln \left(\frac{k \tau_{0}}{2 M}\right)+O\left(k^{-1}\right) .
\end{aligned}
$$

Therefore, to leading order in $k$ and $\epsilon$, we have

$$
\frac{2 \tau_{0}}{\pi} \int_{0}^{\infty} \frac{T_{\mu \nu} u^{\mu} u^{\nu} d \tau}{\tau^{2}+\tau_{0}^{2}} \sim-\frac{1}{24 \pi^{2} \tau_{0} \delta \tau}
$$

where

$$
\delta \tau=\frac{\epsilon}{k}
$$

Note that in these limits $\delta \tau \ll M$ and $\delta \tau \ll \tau_{0}[33]$.

What is the physical significance of $\delta \tau$ ? The negative energy density drops off very rapidly with increasing $r$. In the limit $k \gg 1$ and $\epsilon \ll M$, if we consider the proper time spent by the observer in the region of appreciable negative energy to be

$$
\int_{0}^{\delta \tau} d \tau \sim \frac{1}{k} \int_{2 M+\epsilon}^{2 M+x \epsilon} d r=\frac{1}{k} \epsilon(x-1)
$$

then typically $x-1 \sim O(1)$, so

$$
\delta \tau \sim \frac{\epsilon}{k}
$$

To see this a little more explicitly, note that near the horizon, Eq. (21) becomes

$$
T_{\mu \nu} u^{\mu} u^{\nu} \sim-\frac{k^{2}}{48 \pi(r-2 M)^{2}} .
$$

If the observer starts at $r=r_{0}=2 M+\epsilon$, then at $r=r_{1}=2 M+2 \epsilon$,

$$
\left.\left.\left(T_{\mu \nu} u^{\mu} u^{\nu}\right)\right|_{r=r_{1}} \approx \frac{1}{4}\left(T_{\mu \nu} u^{\mu} u^{\nu}\right)\right|_{r=r_{0}}
$$

so the energy density drops off to $1 / 4$ of its initial value in a distance $\Delta r=\epsilon$, corresponding to a proper time $\delta \tau=\epsilon / k$. Therefore, we should consider the time interval $\delta \tau$ not to be the entire proper time over which the energy density is negative, but rather a time scale which characterizes a change in the energy density.

For an outgoing timelike observer in the Boulware vacuum:

$$
T_{\mu \nu} u^{\mu} u^{\nu}=\frac{1}{24 \pi} C^{-2}\left\{k^{2}\left[\frac{6 M^{2}}{r^{4}}-\frac{4 M}{r^{3}}\right]+\frac{C M^{2}}{r^{4}}\right\} .
$$


Note that this quantity is negative everywhere for $r>2 M$. If we perform the same set of operations as discussed above, we find that Eq. (27) holds for the Boulware vacuum as well. In the $\tau_{0} \rightarrow \infty$ limit of Eq. (27), we "sample" the entire (i.e., half-infinite) geodesic and find

$$
\int_{0}^{\infty} T_{\mu \nu} u^{\mu} u^{\nu} d \tau \approx-\frac{1}{48 \pi \delta \tau},
$$

and hence

$$
\int_{0}^{\infty} T_{\mu \nu} u^{\mu} u^{\nu} d \tau \gtrsim-\frac{1}{\delta \tau}
$$

The dominant contribution to the integral in Eq. (34) is from the lower limit, since $T_{\mu \nu} u^{\mu} u^{\nu}$ in the Boulware vacuum state also has the form given in Eq. (31) near the horizon, but drops off rapidly with increasing $r$. By contrast, the $\tau_{0} \rightarrow \infty$ limit of Eq. (27) for the Unruh vacuum state results in an integral which diverges positively at large $r$. This is due to the fact that in 2D the Hawking radiation does not drop off with distance, but remains constant at large $r$. Therefore, AWEC is satisfied in the Unruh vacuum state in 2D. However, as discussed in Sec. (3), the situation is different in 4D. We could perform the integral on the left-hand-side of Eq. (34) for the Unruh vacuum state, but instead truncate the integration at some finite value of $r=r_{\max }$, with $2 M+\epsilon<r_{\max }<\infty$. For fixed $r_{\max }$, by making $\epsilon$ small enough we can always arrange it so that the negative energy contribution from the lower limit, given by Eq. (31), dominates the integral. In that case, using Eq. (31), we have that

$$
\int_{0}^{\tau\left(r_{\max }\right)} T_{\mu \nu} u^{\mu} u^{\nu} d \tau \approx-\frac{1}{48 \pi \delta \tau} \gtrsim-\frac{1}{\delta \tau}
$$

The inequalities, Eqs. (35) and (36), represent bounds on the degree of AWEC violation seen by timelike geodesic observers who start out very close to the horizon and are shot outward at high speed. In this limit, the longer the (proper) timescale over which the observer sees a significant change in the energy density, the smaller is the magnitude of the integrated negative energy density seen by that observer. Since the negative energy density drops off rapidly with increasing $r$, to remain in the negative energy density the observer must stay close to the horizon. The closer the observer is to the horizon, the larger is the magnitude of the negative energy density. However, in order to remain close to the horizon for a long time as seen by a distant observer, the observer's trajectory must be nearly lightlike. Therefore, although the observer spends a long time in the negative energy region as seen by the distant observer, the proper time spent in the region of appreciable negative energy (as measured by $\delta \tau$ ) decreases with the observer's proximity to the horizon. These inequalities are similar in form to Eq. (6). 


\subsection{Null Geodesics in 2D: Off the Horizon}

We now wish to examine ANEC for null geodesics in 2D Schwarzschild spacetime. From Eq. (13), and from the equation for null geodesics, we have

$$
\frac{d t}{d \lambda}= \pm C^{-1} E, \quad \frac{d r}{d \lambda}= \pm E
$$

where $E$ is an arbitrary positive constant, whose value fixes the scale of the affine parameter, $\lambda$. We want to examine ANEC along both ingoing and outgoing futuredirected (i.e., $d \lambda>0$ ) null geodesics, inside and outside the horizon. There are four cases (see Fig. 1):

$$
\begin{aligned}
& \text { 1) } \frac{d t}{d \lambda}>0, \frac{d r}{d \lambda}=E>0, \quad \text { for } r>2 M \text { (outgoing rays), } \\
& \text { 2) } \frac{d t}{d \lambda}>0, \frac{d r}{d \lambda}=-E<0, \text { for } r>2 M \text { (ingoing rays), } \\
& \text { 3) } \frac{d t}{d \lambda}>0, \quad \frac{d r}{d \lambda}=-E<0, \text { for } r<2 M \text { (outgoing rays), } \\
& \text { 4) } \frac{d t}{d \lambda}<0, \frac{d r}{d \lambda}=-E<0, \text { for } r<2 M \text { (ingoing rays). }
\end{aligned}
$$

Note that $C=(1-2 M / r)$ changes sign inside the horizon, while $d \lambda$ remains positive for future-directed null rays.

If we use Eqs. (38) - (41), and Eqs. (15) - (17), we obtain for the Unruh vacuum state

$$
T_{\mu \nu} K^{\mu} K^{\nu}=\frac{E^{2}}{24 \pi}\left(1-\frac{2 M}{r}\right)^{-2}\left[\frac{6 M^{2}}{r^{4}}-\frac{4 M}{r^{3}}\right] \text { (outgoing) }
$$

and

$$
T_{\mu \nu} K^{\mu} K^{\nu}=\frac{E^{2}}{24 \pi}\left(1-\frac{2 M}{r}\right)^{-2}\left[\frac{6 M^{2}}{r^{4}}-\frac{4 M}{r^{3}}+\frac{1}{8 M^{2}}\right] \text { (ingoing) } .
$$

To obtain Eq. (43), we have used the fact that the sign of $T_{t r}$ changes inside the horizon, as can be seen from Eq. (16), together with Eqs. (39) and (41). Note that for Eq. (43), the term in square brackets vanishes as $r \rightarrow 2 M$. A Taylor expansion shows that, to leading order, it vanishes as $(r-2 M)^{2}$, so it will cancel the $(r-2 M)^{2}$ divergence in the denominator. Therefore Eq. (43) is finite on the horizon, as it should be. However, there appears to be a discontinuity in Eq. (42) for an outgoing null ray on the horizon, $r=2 M$. The situation for null rays on the horizon is sufficiently subtle as to warrant a separate discussion. This is provided in the next subsection.

From Eq. (42), $T_{\mu \nu} K^{\mu} K^{\nu}$ is negative for outgoing null geodesics when $r>1.5 M$. Therefore the local null energy condition, Eq. (2) is violated along outgoing null vectors slightly inside the horizon, as well as along all outgoing rays outside (and on) the horizon. For $r>2 M$, consider an outgoing null geodesic starting at 
$r=2 M+\epsilon$, with $\epsilon \ll M$. Examine ANEC along this ray to obtain

$$
I_{1} \equiv \int_{0}^{\infty} T_{\mu \nu} K^{\mu} K^{\nu} d \lambda=\frac{E}{24 \pi} \int_{2 M+\epsilon}^{\infty} \frac{1}{(r-2 M)^{2}}\left[\frac{6 M^{2}}{r^{2}}-\frac{4 M}{r}\right] d r
$$

where we have used Eq. (38). Here $\lambda=0$ is the value of the affine parameter at $r=2 M+\epsilon$. Equations (42) and (44) apply for the Boulware vacuum as well as for the Unruh vacuum. This is due to the fact that in the latter case, the outgoing null geodesics are parallel to the Hawking radiation. Hence the terms which would cause the integral in Eq. (36) to diverge as $r_{\max } \rightarrow \infty$ are absent in the null geodesic case.

For $r<2 M$, along an outgoing null geodesic starting just inside the horizon at $r=2 M-\epsilon$ :

$$
I_{2} \equiv \int_{0}^{\lambda_{f}} T_{\mu \nu} K^{\mu} K^{\nu} d \lambda=\frac{E}{24 \pi} \int_{r_{\min }}^{2 M-\epsilon} \frac{1}{(r-2 M)^{2}}\left[\frac{6 M^{2}}{r^{2}}-\frac{4 M}{r}\right] d r
$$

where we have used Eq. (40), and $\lambda_{f}$ is the value of the affine parameter at $r=r_{\text {min }}$, the minimum value of $r$ attained by the null geodesics. Notice that the integrand in Eq. (45) will be dominated by the positive $6 M^{2} / r^{2}$ term which diverges for small $r$. From Eq. (43), we see that $T_{\mu \nu} K^{\mu} K^{\nu}$ is positive everywhere for ingoing null geodesics. Thus we immediately see that ANEC is satisfied for these two sets of null geodesics.

In Eq. (44) we perform the integration and then expand the result in the limit of small $\epsilon$ to find

$$
\lim _{\epsilon \rightarrow 0} I_{1} \sim-\frac{E}{48 \pi \epsilon}+O(\log (\epsilon / M))<0 .
$$

Note that $\delta \lambda=E / \epsilon$ is the characteristic affine parameter distance over which the negative energy is decreasing along the outgoing geodesic. We can rewrite Eq. (46) as

$$
\int_{0}^{\infty} T_{\mu \nu} K^{\mu} K^{\nu} d \lambda \approx-\frac{1}{48 \pi \delta \lambda} \gtrsim-\frac{1}{\delta \lambda}
$$

This is the null version of Eq. (36). Note that it is invariant under rescaling of the affine parameter.

Similarly, for Eq. (45), we may perform the integration and expand the result in the small $r_{\min }$ limit. For fixed $\epsilon$, the result is

$$
\lim _{r_{\min } \rightarrow 0} I_{2} \sim \frac{E}{16 \pi r_{\min }}+O\left(\ln \left(r_{\min } / M\right)\right)>0
$$

However, for fixed $r_{\text {min }}$ and $\epsilon \rightarrow 0, I_{2}$ has the same behavior as $I_{1}$ :

$$
I_{2} \sim-\frac{E}{48 \pi \epsilon}+O(\ln (\epsilon / M))<0
$$

Thus we see that ANEC is violated for outgoing null rays just outside the horizon, but it is satisfied for outgoing null rays inside the horizon in the limit $r_{\min } \rightarrow 0$. (The 
divergence in Eq. (46) as $\epsilon \rightarrow 0$ may be circumvented by an appropriate choice of scaling of the affine parameter, i.e., by an appropriate choice of $E$. This and related issues will be discussed in the next sub-section.) Outgoing rays which originate in the region $1.5 M<r<2 M$ initially encounter negative energy, and later encounter positive energy near the singularity, $r=0$. This is analogous to the case of outgoing timelike geodesics in the Unruh vacuum, which encounter negative energy near the horizon and positive energy at large distances due to the Hawking radiation. We also see that ANEC holds for ingoing null geodesics, whether they originate outside or inside the horizon.

One might at first sight conclude that the reasoning here is a bit circular. It could be argued that it is no surprise that evaluation of the ANEC integral for null geodesics inside the horizon yields a divergent positive result. After all, we placed our quantized field on a background which already had a singularity in it. Thus one could argue that divergent values of our ANEC integrals for these geodesics are not unexpected, because the behavior of the fields inside the horizon is dominated by the singularity. However, it is not obvious, a priori, what the sign of these integrals should be. Since these are test fields on a given background, as opposed to fields which generate the background spacetime (which we cannot have in any case in 2D), it is possible that the integrals might have turned out to diverge with either sign. Similar ANEC integrals for quantized massless fermion fields in 2D have the same signs as for the massless scalar field. This follows from the fact that the renormalized fermion stress tensor is identical to that in the scalar case [34], even though the formally divergent tensors have opposite signs in the two cases.

\subsection{Null Geodesics in 2D: On the Horizon}

Recall that our results for outgoing null geodesics outside the horizon are formulated in terms of the null tangent vector

$$
K^{\mu}=E\left(C^{-1}, 1\right)
$$

where the components are given in terms of Schwarzschild $(t, r)$ coordinates. Since these coordinates are badly-behaved on the horizon, let us switch to Kruskal null coordinates, given by

$$
U=-e^{-\kappa u}, \quad V=e^{\kappa v}
$$

where $u=t-r^{*}, v=t+r^{*}$, with $r^{*}$ the usual tortoise coordinate and $\kappa=1 /(4 M)$. In these coordinates, our null vector has the form

$$
K^{\bar{\mu}}=\left(\frac{d U}{d \lambda}, \frac{d V}{d \lambda}\right)=\left(0, \frac{2 E}{C} \kappa e^{\kappa v}\right) .
$$

If $E$ has the same value for all outgoing null geodesics, then $K^{\bar{\mu}}$ would not be defined in the limit where the geodesics approach the horizon, since $C \rightarrow 0$. However, $E$ need 
only be constant along each null geodesic, but not the same constant for different null geodesics.

Consider a sequence of outgoing null geodesics which start at different values of $r$, e.g., the histories of a sequence of photons emitted by an infalling observer. Let $r_{0}$ be the value of $r$ at which the null geodesic begins (which will be different for each null geodesic). We might choose the various values of $E$ for different null geodesics by the following prescription. Let the observer emit photons of fixed frequency $\omega_{e}$ in his rest frame. The time-component of the observer's two-velocity (in Schwarzschild coordinates) is

$$
u^{t}=\frac{d t}{d \tau}=\left(1-\frac{2 M}{r_{0}}\right)^{-1} \tilde{E},
$$

where $\tilde{E}$ is the observer's energy per unit rest mass, measured at infinity. This is just the rate of a clock at infinity as compared to the rate of the clock carried by the infalling observer. The frequency of a photon at infinity is $\omega_{\infty}$, which is related to $\omega_{e}$ by

$$
\frac{\omega_{\infty}}{\omega_{e}}=\frac{d \tau}{d t}=\left(1-\frac{2 M}{r_{0}}\right) \tilde{E}^{-1} .
$$

Note that the expression on the right-hand-side includes both the Doppler effect and the gravitational redshift. The factor of $\tilde{E}^{-1}$ reflects the fact that the faster the observer is shot toward the black hole (i.e., the larger $\tilde{E}$ is), the greater will be the Doppler shift. If we choose a scaling for the affine parameter such that $E=\omega_{\infty}$, for fixed $\omega_{e}$, then

$$
E=\left(1-\frac{2 M}{r_{0}}\right) \omega_{e} \tilde{E}^{-1} \propto C\left(r_{0}\right) .
$$

Now $E / C\left(r_{0}\right)$ is finite in the limit $r_{0} \rightarrow 2 M$, so that the components of $K^{\mu}$ are finite on the horizon, in either Schwarzschild or Kruskal coordinates.

However, $K^{\bar{\mu}}$ is still not the same as $k^{\bar{\mu}}$, the affinely parameterized null tangent vector on the horizon. In Kruskal null coordinates the vector $k^{\bar{\mu}}$ has components

$$
k^{\bar{\mu}}=(0, \kappa)
$$

(See the discussion on p. 331 of Ref. [35].) On the horizon,

$$
K^{\bar{\mu}}=\left(\frac{2 E}{C}\right)_{r=2 M} e^{\kappa v}(0, \kappa),
$$

so these vectors still differ by a factor of $\exp (\kappa v)$. Thus, a null vector such as $K^{\mu}$, which is an affinely parameterized null tangent vector off the horizon, does not necessarily remain affinely parameterized on the horizon.

With these lessons in hand, let us now consider the integral $\int T_{\bar{\mu} \bar{\nu}} k^{\bar{\mu}} k^{\bar{\nu}} d V$ along a portion of the future event horizon. Here $T_{\bar{\mu} \bar{\nu}}$ is the vacuum expectation value of the stress-tensor in the Unruh vacuum state expressed in Kruskal null coordinates, 
and $k^{\bar{\mu}}$ is an affinely parameterized null tangent vector on the horizon. The Kruskal advanced time coordinate $V$, is an affine parameter on the horizon (see for example, p. 331 of Ref. [35 or p. 122 of Ref. [36]). From Eq. (56), and a straightforward coordinate transformation of Eqs. (15)- (17), we obtain

$$
\int_{V_{0}}^{\infty} T_{\bar{\mu} \bar{\nu}} k^{\bar{\mu}} k^{\bar{\nu}} d V=-\frac{1}{768 \pi M^{2} V_{0}} .
$$

This integral is taken over a portion of the future horizon; $V_{0}$ is the value of the $V=$ const line which intersects the future horizon at the (arbitrary) event where we start our null geodesic.

The right-hand-side of Eq. (58) goes to $-\infty$ as $V_{0} \rightarrow 0$. However, this would correspond to integrating along the full future horizon of an eternal black hole spacetime. The Unruh vacuum state is singular on the past horizon (i.e., $V=0$ ) of such a spacetime, so it is not surprising that we get a divergence in this case. Physically, this would correspond to a black hole that Hawking-radiates for an infinite time. For the Unruh vacuum state to be realizable, we must consider only physically realistic collapse spacetimes in which the black hole forms at a finite time in the past. Therefore, the smallest allowed value of $V_{0}=V_{\text {min }}$, which is the value of the $V=$ const line at which the the worldline of the surface of the collapsing star intersects the future event horizon, as shown in Fig. (2). For such spacetimes, the ANEC integral along a portion of the future horizon is negative but finite. This result seems to be in agreement with the results of Wald and Yurtsever 114. They find that ANEC is satisfied along the complete null geodesic comprised of the future horizon plus the null line which would have been the past horizon in an eternal black hole spacetime. In a collapse spacetime, this complete null geodesic originates at past null infinity, propagates through the collapsing star, "reflects off" $r=0$, and then emerges into the vacuum outside the star at $V_{0}=V_{\min }$ (see Fig. (2)). Evidently there is a positive contribution to the ANEC integral from the part of the geodesic prior to its exit from the collapsing star [37]. This contribution must be greater than or equal to $\left(768 \pi M^{2} V_{\min }\right)^{-1}$. (Note that the source of the positive contribution we refer to here is the quantized field, not the classical collapsing matter. The latter would presumably make the ANEC integral even more positive.)

The dimensions of $k^{\bar{\mu}}$ are (length) ${ }^{-1}$, whereas $T_{\bar{\mu} \bar{\nu}}, V$, and $V_{0}$ are dimensionless. Let us now rescale the coordinates to get a more familiar choice of dimensions. Let $x^{\hat{\mu}}=\kappa^{-1} x^{\mu}$, i.e., $\hat{U}=\kappa^{-1} U, \hat{V}=\kappa^{-1} V$, so $\hat{V}_{0}=\kappa^{-1} V_{0}$. Then $T_{\hat{\mu} \hat{\nu}}=\kappa^{2} T_{\bar{\mu} \bar{\nu}}$, and $k^{\hat{\mu}}=\kappa^{-1} k^{\bar{\mu}}$. If we rewrite Eq. (58) in terms of the rescaled coordinates, we obtain

$$
\begin{aligned}
\int_{\hat{V}_{0}}^{\infty} T_{\hat{\mu} \hat{\nu}} k^{\hat{\mu}} k^{\hat{\nu}} d \hat{V} & =-\frac{1}{768 \pi \kappa^{2} M^{2} \hat{V}_{0}} \\
& =-\frac{1}{48 \pi \hat{V}_{0}} .
\end{aligned}
$$

Compare this result with our result for null rays outside the horizon, i.e., Eq. (47), in which $\delta \lambda$ is interpreted as the characteristic affine parameter distance along the 
null geodesic over which the negative energy density falls off. Let us now show that a similar interpretation holds on the horizon. The integrand of Eq. (59) is equal to $-1 /\left(48 \pi \hat{V}^{2}\right)$, so

$$
\begin{aligned}
\int_{\hat{V}_{0}}^{\infty} T_{\hat{\mu} \hat{\nu}} k^{\hat{\mu}} k^{\hat{\nu}} d \hat{V} & =-\frac{1}{48 \pi} \int_{\hat{V}_{0}}^{\infty}\left(\frac{1}{\hat{V}^{2}}\right) d \hat{V} \\
& =-\frac{1}{48 \pi \hat{V}_{0}} .
\end{aligned}
$$

Note that $\hat{V}_{0}$ can be interpreted as the characteristic affine parameter distance over which the integrand falls off, i.e., as $\hat{V}$ increases from $\hat{V}=\hat{V}_{0}$ to $\hat{V}=2 \hat{V}_{0}, T_{\hat{\mu} \hat{\nu}} k^{\hat{\mu}} k^{\hat{\nu}}$ falls off to $1 / 4$ of its initial value. Thus the interpretation of the QI for null geodesics on the horizon is the same as that for Eq. (47).

\section{4D Evaporating Black Holes}

In this section, we examine AWEC and ANEC in four-dimensional evaporating black hole spacetimes. In these spacetimes, the components of the renormalized expectation value of the stress-energy tensor are only known numerically. Accordingly, we make use of the numerical data of Elster [38 for the conformally-coupled massless scalar field and that of Jensen, McLaughlin, and Ottewill [39] for the electromagnetic field, evaluated in the Unruh vacuum state. We examine the cases of outgoing radial timelike observers, outgoing radial null geodesics, and orbiting null geodesics. Only geodesics outside the horizon are considered, as no numerical data are available for $r \leq 2 M$. We find that although AWEC and ANEC are violated in all three cases, there appear to be QI-type bounds in each case which constrain the degree of the violations, as in two-dimensional spacetime.

These results might at first sight seem to be in disagreement with recent results of Visser [40]. He found that for the renormalized vacuum expectation value of the stress-energy tensor of a conformally-coupled massless scalar field in Schwarzschild spacetime, ANEC is satisfied for all null geodesics that reach spatial infinity, and for orbiting geodesics at $r=3 M$. However, his results are applicable for the HartleHawking vacuum state, which represents a black hole in thermal equilibrium with a surrounding external thermal bath of radiation. It is therefore not too surprising to

find that ANEC holds in that case. By contrast, our analysis is performed in the Unruh vacuum state, which corresponds to the situation of a black hole evaporating into empty space.

\subsection{Outgoing Radial Timelike Observers}

The four-velocity of an outgoing radial timelike observer in 4D Schwarzschild spacetime is given by

$$
u^{\mu}=\left(\frac{k}{C}, \sqrt{k^{2}-C}, 0,0\right)
$$


where again $C=(1-2 M / r)$. Let us adopt the notation of Elster [38] so that: $\mu(r)=-T^{t}{ }_{t}, \quad p_{r}(r)=T_{r}^{r}, \quad L=-4 \pi r^{2} C T_{t r}$, where the components of the stresstensor here represent renormalized expectation values in the Unruh vacuum state. Therefore, we may write

$$
T_{\mu \nu} u^{\mu} u^{\nu}=\frac{k^{2}}{C}\left(\mu+p_{r}\right)-p_{r}-\frac{k L}{2 \pi r^{2} C^{2}} \sqrt{k^{2}-C} .
$$

If a QI-type bound of the kind found in 2D, i.e., Eq. (35), also holds in 4D, then we might expect it [1] to have the form

$$
\int_{0}^{\infty} T_{\mu \nu} u^{\mu} u^{\nu} d \tau \gtrsim-\frac{1}{M^{2} \delta \tau},
$$

where again $\delta \tau$ is the characteristic proper time which the observer spends in the negative energy. (Note that since the fields we will be considering are massless, the mass $M$ of the black hole is only natural length scale which appears in the problem.) We want to numerically compare the left and right-hand-sides of Eq. (63), in order to see if the inequality is satisfied.

The integrand in Eq. (63) drops off rapidly with increasing $r$ near the horizon (unlike in the 2D case), hence the dominant contribution to the AWEC integral comes from the initial part of the trajectory. We will therefore approximate the AWEC integral as

$$
\left.\int_{0}^{\infty} T_{\mu \nu} u^{\mu} u^{\nu} d \tau \approx T(r)\right|_{r_{0}} \delta \tau
$$

where $T(r) \equiv T_{\mu \nu} u^{\mu} u^{\nu}$ is understood to be evaluated at the starting point $r_{0}$ (minimum $r$ ) of the trajectory. Since the magnitude of $T(r)$ rapidly decreases near the horizon, our estimate of using $T\left(r_{0}\right)$ gives a result which is somewhat more negative than the true result. Therefore, correction of this estimate only goes in the direction of strengthening the inequality.

Our results for the electromagnetic field are displayed in Table 1. The values of $\mu$ and $p_{r}$ for the electromagnetic field are from the numerical data given in Ref. [39], and the value of $L=3.4 \times 10^{-4} / M^{2}$ used in this case is the photon luminosity given by Page [42. In the fourth column of Table $1, \delta r$ is chosen such that the magnitude of $T(r)$ decreases by at least a factor of two from $r_{0}$ to $r_{0}+\delta r$. The characteristic proper time, $\delta \tau$, is computed from

$$
\delta \tau=\int_{r_{0}}^{r_{0}+\delta r} \frac{d r}{\sqrt{k^{2}-C}} .
$$

The next to last column in the table is our approximation of the AWEC integral given in Eq. (64), while the last column represents the right-hand-side of our QI given by Eq. (63). By examining the last two columns of Table 1, we see that the QI, Eq. (63), is easily satisfied. A similar analysis for the conformally-coupled massless scalar field shows that the QI is easily satisfied in that case as well. Numerical values of $\mu, p_{r}$, and $L=7.44 \times 10^{-5} / M^{2}$ for the scalar field were obtained from the numerical data given in Ref. 38. 


\begin{tabular}{|c|c|c|c|c|c|c|c|}
\hline$r_{0}$ & $\mu\left(r_{0}\right) M^{4}$ & $p_{r}\left(r_{0}\right) M^{4}$ & $\delta r / M$ & $k$ & $\delta \tau / M$ & $T \delta \tau$ & $-\left(M^{2} \delta \tau\right)^{-1}$ \\
\hline \multirow[t]{4}{*}{2.1} & $-2.7 \times 10^{-3}$ & $1.9 \times 10^{-3}$ & 0.5 & 0.5 & 1.77 & $-1.1 \times 10^{-2}$ & -0.56 \\
\hline & & & & 1.0 & 0.54 & $-1.0 \times 10^{-2}$ & -1.85 \\
\hline & & & & 5.0 & 0.10 & $-4.4 \times 10^{-2}$ & -10 \\
\hline & & & & 10.0 & 0.05 & $-8.5 \times 10^{-2}$ & -20 \\
\hline \multirow[t]{3}{*}{2.2} & $-1.8 \times 10^{-3}$ & $1.5 \times 10^{-3}$ & 1.0 & 1 & 1.16 & $-5.7 \times 10^{-3}$ & -0.86 \\
\hline & & & & 5 & 0.20 & $-1.7 \times 10^{-2}$ & -5 \\
\hline & & & & 10 & 0.10 & $-3.5 \times 10^{-2}$ & -10 \\
\hline \multirow[t]{3}{*}{2.5} & $-1.1 \times 10^{-3}$ & $6.8 \times 10^{-4}$ & 1.0 & 1 & 1.22 & $-3.3 \times 10^{-3}$ & -0.82 \\
\hline & & & & 5 & 0.20 & $-1.0 \times 10^{-2}$ & -5 \\
\hline & & & & 10 & 0.10 & $-2.1 \times 10^{-2}$ & -10 \\
\hline \multirow[t]{3}{*}{3} & $-5.4 \times 10^{-4}$ & $3.2 \times 10^{-4}$ & 2 & 1 & 2.82 & $-2.8 \times 10^{-3}$ & -0.45 \\
\hline & & & & 5 & 0.40 & $-6.8 \times 10^{-3}$ & -2.5 \\
\hline & & & & 10 & 0.20 & $-1.3 \times 10^{-2}$ & -5 \\
\hline
\end{tabular}

Table 1: Outgoing timelike geodesics moving through the quantized electromagnetic field.

\subsection{Radial Null Rays}

The components of the tangent vector to an affinely parameterized outgoing radial null geodesic (outside the horizon) are given by

$$
K^{\mu}=E\left(C^{-1}, 1,0,0\right) .
$$

Thus we have

$$
T_{\mu \nu} K^{\mu} K^{\nu}=\frac{E^{2}}{2 \pi(r-2 M)^{2}}\left\{2 \pi r(r-2 M)\left[\mu(r)+p_{r}(r)\right]-L\right\} .
$$

The 4D analog of the QI for null geodesics in 2D given by Eq. (47) is

$$
\int_{0}^{\infty} T_{\mu \nu} K^{\mu} K^{\nu} d \lambda \gtrsim-\frac{1}{M^{2} \delta \lambda},
$$

where $\delta \lambda$ is again interpreted as the characteristic affine parameter distance along the null geodesic over which the negative energy density falls off. If we define

$$
T \equiv \frac{1}{E^{2}}\left(T_{\mu \nu} K^{\mu} K^{\nu}\right),
$$

and use the fact that $d r / d \lambda=E$ and $\delta \lambda=\delta r / E$, then it is easily seen that the QI, Eq. (68) is equivalent to

$$
\int_{r_{0}}^{\infty} T d r \gtrsim-\frac{1}{M^{2} \delta r}
$$

where $r_{0}>2 M$ is the initial starting value of $r$ for the outgoing null geodesic and $\delta r$ is the characteristic interval in $r$ over which the negative energy falls off. We numerically 


\begin{tabular}{|r|r|r|r|r|r|}
\hline$r_{0} / M$ & $\mu\left(r_{0}\right) M^{4}$ & $p_{r}\left(r_{0}\right) M^{4}$ & $\delta r / M$ & $T \delta r M^{3}$ & $-M / \delta r$ \\
\hline 2.5 & $-4 \times 10^{-6}$ & 0 & 0.5 & $-3.4 \times 10^{-5}$ & -2 \\
4 & $-4 \times 10^{-7}$ & $-3 \times 10^{-7}$ & 3 & $-4.8 \times 10^{-6}$ & -0.3 \\
5 & $-1.5 \times 10^{-6}$ & $-3 \times 10^{-7}$ & 5 & $-7.8 \times 10^{-6}$ & -0.2 \\
\hline
\end{tabular}

Table 2: Data is given for outgoing null geodesics, where $\mu$ and $p_{r}$ are the energy density and pressure for a massless scalar field.

\begin{tabular}{|r|r|r|r|r|r|}
\hline$r_{0} / M$ & $\mu\left(r_{0}\right) M^{4}$ & $p_{r}\left(r_{0}\right) M^{4}$ & $\delta r / M$ & $T \delta r M^{3}$ & $-M / \delta r$ \\
\hline 2.1 & $-2.7 \times 10^{-3}$ & $1.9 \times 10^{-3}$ & 0.5 & -0.01 & -2 \\
2.2 & $-1.8 \times 10^{-3}$ & $1.5 \times 10^{-3}$ & 1 & -0.004 & -1 \\
2.5 & $-1.1 \times 10^{-3}$ & $6.8 \times 10^{-4}$ & 1 & -0.002 & -1 \\
3 & $-5.4 \times 10^{-4}$ & $3.2 \times 10^{-4}$ & 2 & $-1.4 \times 10^{-3}$ & -0.5 \\
\hline
\end{tabular}

Table 3: Data is given for outgoing null geodesics, where $\mu$ and $p_{r}$ are the energy density and pressure for the electromagnetic field.

evaluate each side of Eq. (70) to see if the inequality is satisfied. Following a similar procedure to that used for outgoing radial timelike observers, we approximate the ANEC integral by

$$
\int_{r_{0}}^{\infty} T d r \approx T\left(r_{0}\right) \delta r
$$

As discussed in the last section, this approximation gives a result which is somewhat more negative than the true result. Therefore, if the QI holds for our approximation, then the inequality in the actual case is even stronger.

Our results for the scalar field are given in Table 2, and those for the electromagnetic field appear in Table 3, where as before the numerical data of Refs. [38] and [39], were used for the scalar and electromagnetic fields, respectively. The next to last column in each table is our approximation of the ANEC integral given in Eq. (71), while the last column represents the right-hand-side of our QI given by Eq. (70). By examining the last two columns of each table, we see that the QI, Eq. (70), is easily satisfied and hence so is Eq. (68). Note that the inequalities in $4 \mathrm{D}$ seem to be satisfied by a wider margin than the corresponding QI's in 2D. This is due to the fact that the numerical factors which appear in the components of the renormalized expectation values of the stress-tensor are characteristically smaller in $4 \mathrm{D}$ than in $2 \mathrm{D}$ spacetime, at least for radial null geodesics in the scalar field case.

In our calculations, we have ignored the backreaction of the Hawking radiation on the background spacetime. It might be better to repeat our calculations on an evolving background, such as a Vaidya spacetime. However, we can estimate the effects of backreaction on an outgoing null ray just outside the horizon in the following way. Our approximation should hold as long as the time of escape of a null ray from near the horizon is small compared to the evaporation time of the black hole. Here 
these times are measured by an observer at infinity. For such a null ray we have

$$
\int_{0}^{t_{e s c}} d t=\int_{2 M+\epsilon}^{x M}(1-2 M / r)^{-1} d r
$$

Solving for $t_{e s c}$ yields

$$
t_{e s c}=x M+2 M \ln \left(\frac{x}{2}-1\right)-2 M-\epsilon+2 M \ln \left(\frac{2 M}{\epsilon}\right),
$$

where $t_{\text {esc }}$ is the time for the ray to go from $r=2 M+\epsilon$ to $r=x M$. For $x \gg 2$ and finite, in the limit of small $\epsilon$ :

$$
t_{\text {esc }} \rightarrow 2 M \ln \left(\frac{2 M}{\epsilon}\right)
$$

By contrast, the evaporation time of the hole is $t_{\text {evap }}=A M^{3}$, where $A \approx 10^{4} / n$, and $n$ is the number of species of particles, each of which is assumed to contribute approximately $10^{-4} M^{-2}$ to the black hole's luminosity. Our approximation should break down only when $\epsilon \lesssim 2 M \exp \left[-A M^{2} / 2\right]$.

\subsection{Orbiting Null Rays}

We now examine orbiting null geodesics in Schwarzschild spacetime. The line element is

$$
d s^{2}=-C d t^{2}+C^{-1} d r^{2}+r^{2}\left(d \theta^{2}+\sin ^{2} \theta d \phi^{2}\right),
$$

where again $C=(1-2 M / r)$. For an orbiting null ray in Schwarzschild with $\theta=$ $\pi / 2=$ const

$$
\frac{d t}{d \lambda}=\frac{r}{\sqrt{C}} \frac{d \phi}{d \lambda}
$$

Here we choose $d \phi / d \lambda>0$, for our future-directed ray. From the geodesic equations, we have

$$
\begin{aligned}
\dot{t} & \equiv \frac{d t}{d \lambda}=\frac{E}{C} \\
\dot{\phi} & \equiv \frac{d \phi}{d \lambda}=\frac{L}{r^{2}}
\end{aligned}
$$

where $E$ and $L$ are constants. It follows that

$$
\frac{L}{E}=r C^{-1 / 2}
$$

At $r=3 M$, we have

$$
\left.\frac{L}{E}\right|_{r=3 M}=3 \sqrt{3} M
$$


From the numerical values in Figs. (1) and (3) of Ref. [39 for the quantized electromagnetic field, and Eq. (80), we find that

$$
\begin{aligned}
\left.T_{\mu \nu} K^{\mu} K^{\nu}\right|_{r=3 M} & =\left.3 E^{2}\left[-T_{t}^{t}+T_{\phi}^{\phi}\right]\right|_{r=3 M} \\
& \approx-3.1 \times 10^{-4} \frac{E^{2}}{M^{4}},
\end{aligned}
$$

where $K^{\mu}=d x^{\mu} / d \lambda$ is the tangent to the null geodesic. For one orbit, $\Delta \phi=2 \pi=$ $\left(L / 9 M^{2}\right) \Delta \lambda$, so

$$
\frac{E}{M}=\frac{2 \sqrt{3} \pi}{\Delta \lambda}
$$

We may therefore rewrite Eq. (81) as

$$
\left.T_{\mu \nu} K^{\mu} K^{\nu}\right|_{r=3 M} \approx-3.7 \times 10^{-2} \frac{1}{M^{2}(\Delta \lambda)^{2}}
$$

Integration of $T_{\mu \nu} K^{\mu} K^{\nu}$ over one orbit gives

$$
\int_{0}^{\Delta \lambda} T_{\mu \nu} K^{\mu} K^{\nu} d \lambda \approx-3.7 \times 10^{-2} \frac{1}{M^{2}(\Delta \lambda)}
$$

Thus we see that for orbiting null geodesics

$$
\int_{0}^{\Delta \lambda} T_{\mu \nu} K^{\mu} K^{\nu} d \lambda \gtrsim-\frac{1}{M^{2}(\Delta \lambda)}
$$

The expressions given by Eqs. (84) and (85) are of the same form as those for radial null geodesics discussed earlier. The difference here is that we integrate only over one orbit, i.e., we do not consider multiple traversals through the same negative energy region. The inclusion of the contributions of an arbitrary number of orbits would violate the inequality given in Eq. (85). (Since the $r=3 M$ orbit is unstable, realistically we would expect the null ray to at best only make a few orbits.) However, it is of interest to note that if we consider the null geodesics which generate the boundary of the future of an event $P$ with $r=3 M$, then the orbiting null geodesics leave this boundary after half an orbit. This is due to the fact that orbiting null geodesics originating at $P$, which initially set off in opposite spatial directions, encounter a conjugate point (i.e., the geodesics cross) on the far side of the black hole after half an orbit [43], 44]. At this point, the geodesics leave the boundary of the future of $P$ (i.e., an event $Q$ which lies beyond the conjugate point along either null geodesic can be connected to $P$ by a timelike curve), and therefore are not achronal.

This observation may be relevant in the context of proving singularity theorems in the presence of negative energy. For example, in the proof of a singularity theorem such as the Penrose theorem, one uses either the local or the averaged null energy condition to insure the focusing of the null geodesics which generate the boundary of 
the future of a closed trapped surface. Geodesics of the orbiting variety considered here would not relevant, at least in the spherically symmetric case, since they leave the boundary of the future of a point in any case, irrespective of energy conditionfocusing arguments. We also note in passing that, at least in this simple case, while these null geodesics are in the boundary they obey the inequality, Eq. (85).

Let us compare the previous results with the following situation. Consider a 4D Minkowski spacetime with one spatial dimension compactified, and a quantized, minimally-coupled, massless scalar field in the Casimir vacuum state on this background. Let $K^{\mu}=E(1,1,0,0)$ be the tangent to a null geodesic in the $x$-direction. The vacuum expectation value of the stress-tensor in this state is

$$
T_{\mu \nu}=\frac{\pi^{2}}{45 L^{4}} \times \operatorname{diag}\{-1,-3,1,1\}
$$

where we have taken $x$ as the compactified dimension with circumference $L$. It follows that

$$
T_{\mu \nu} K^{\mu} K^{\nu}=-\frac{4 \pi^{2} E^{2}}{45 L^{4}} .
$$

The affine parameter lapse for one orbit is $\lambda_{p}=L / E$. If we integrate over one orbit, we find that

$$
\begin{aligned}
\int_{0}^{\lambda_{p}} T_{\mu \nu} K^{\mu} K^{\nu} d \lambda & =\left(T_{\mu \nu} K^{\mu} K^{\nu}\right)\left(\lambda_{p}\right) \\
& =-\frac{4 \pi^{2}}{45 L^{2} \lambda_{p}}
\end{aligned}
$$

We see that this result has the same form as Eq. (84) for the black hole case. Both of these expressions are invariant under rescaling of the affine parameter (as is Eq. (68) for radial null geodesics). Here, as in the black hole case, we have ignored the possibility of multiple orbits, which would violate the inequality. However, it is possible that in the 4D compactified case, as was found previously in 2D [20], an ANEC-type bound might hold on the difference between the expectation values of $T_{\mu \nu} K^{\mu} K^{\nu}$ in an arbitrary quantum state and in the Casimir vacuum state.

\section{Conclusions}

In this paper, we have examined AWEC and ANEC in both two and four- dimensional evaporating Schwarzschild black hole spacetimes, for quantized massless scalar and electromagnetic fields. Our 2D results are the following: It was discovered that AWEC is satisfied for outgoing radial timelike geodesics which start just outside the horizon and reach infinity, for the Unruh vacuum state. This is due to the positive contribution to the AWEC integral of the Hawking radiation at large distances, which outweighs the contribution of the negative energy near the horizon. By contrast, AWEC is violated for such geodesics in the Boulware vacuum state. Similarly, ANEC 
is violated for half-complete outgoing radial null geodesics, which start just outside the horizon, in both the Unruh and Boulware vacuum states. This is also true on the horizon in the Unruh vacuum state. However, ANEC is satisfied along ingoing null geodesics and along outgoing null geodesics inside the horizon in the Unruh vacuum state, due to a large positive contribution to the integral near the singularity.

In $4 \mathrm{D}$, we examined AWEC and ANEC in the Unruh vacuum state, using previously calculated numerical values of the stress-tensor components. There it was found that AWEC is violated for outgoing timelike geodesics which start just outside the horizon and reach infinity. (Note that in 4D, the Hawking radiation decays like $1 / r^{2}$, so the large positive contribution to the AWEC integral found in $2 \mathrm{D}$ does not arise here.) Similarly, we found that ANEC is violated for analogous outgoing null geodesics. This condition is violated for orbiting null geodesics as well.

However, in all cases where the conditions are violated, there appear to be QI-type bounds which limit the degree of the violations. Let $\delta \tau$ be the characteristic proper time for timelike geodesics, and $\delta \lambda$ be the affine parameter length for null geodesics, over which the negative energy density changes significantly. In $2 \mathrm{D}$ the QI bounds have the form that the integrated negative energy density along the timelike or null geodesic (i.e., the AWEC or ANEC integral) is greater than or equal to minus the inverse of $\delta \tau$ or $\delta \lambda$, respectively. In $4 \mathrm{D}$, the corresponding inequalities have additional factors of $M^{-2}$ on the right-hand-sides.

Can the semi-classical effects of negative energy, in processes such as black hole evaporation, invalidate the singularity theorems before quantum gravity effects become significant? Although we do not have a definite proof in the general case, our results suggest that the answer may be no. It remains possible that even though ANEC (or even a weaker energy condition [17]) might fail in some regions of a given spacetime, it may hold in enough other regions for the conclusions of the singularity theorems to be maintained. Our (admittedly) 2D results for ANEC would seem to support this view. In Ref. [9], it was shown that if ANEC is satisfied along the null geodesics which generate the boundary of the future of a trapped surface, then Penrose's singularity theorem will still hold. There the local null energy condition was replaced by ANEC along half-complete null geodesics. However, two points should be emphasized about that result. First, it is not necessary to assume that the ANEC integral is non-negative to prove the result [9, 17]. In fact, from Lemma 3 of Ref. [6] or Eq. (5) of Ref. [9] and the Einstein equations, it is sufficient to have

$$
\int_{0}^{\infty} T_{\mu \nu} K^{\mu} K^{\nu} d \lambda \geq \frac{\theta_{0}}{2}
$$

where $\theta_{0}<0$ is the initial expansion of the null geodesic congruence at the trapped surface. The ANEC integral may be negative as long as it is not more negative than $\theta_{0} / 2$. Physically this implies that a singularity will still form provided that the defocusing effects due to the presence of any negative energy are more than compensated for by the initial convergence of the null geodesics produced by the trapped surface. 
Second, the existence of only one such trapped surface whose orthogonal null geodesics obey either ANEC or the weaker inequality, Eq. (89), is required to prove the occurrence of a singularity. There may exist other trapped surfaces in the spacetime whose orthogonal null geodesics do not obey either of the inequalities. However, as long as there exists at least one trapped surface with the desired properties, a singularity is inevitable.

Consider an evaporating black hole spacetime, with backreaction included. The Penrose diagram for the standard scenario is shown in Fig. (3). Let us assume that this standard picture is correct. The line $\mathrm{H}^{+}$is the event horizon, the dashed curve $A$ is the apparent horizon, and the lines $g_{1}, g_{2}$, and $g_{3}$ are outgoing null geodesics orthogonal to the trapped surfaces labelled 1,2, and 3, respectively. The outgoing null rays from 1 do not focus but instead reach future null infinity, so one expects that any averaged energy condition which would insure focusing, such as ANEC or Eq. (89), would fail along them. The closest analogs to these rays in our analysis are the outgoing null rays just outside the horizon shown in Fig. (1). It was found that in both two and four-dimensional Schwarzschild spacetime, ANEC was violated along these rays, although in each case a QI limits the degree of the violation. One would expect that if an analogous QI holds in more general cases, such as Fig. (3), it will not be strong enough to guarantee focusing of null rays in the region between $H^{+}$and $A$ in Fig. (3). We could not examine the weaker inequality Eq. (89), since in a static evaporating black hole background there are no trapped surfaces outside the horizon. For the outgoing null rays from 2 in Fig. (3) (a trapped surface inside the collapsing matter), one would suspect that ANEC probably holds. In this case, the focusing effects of the matter of the star most likely overwhelm any defocusing by negative energy. For the outgoing null rays from 3 (a trapped surface "behind" the event horizon, in the vacuum outside the collapsing matter), one might expect either ANEC or a similar but weaker inequality such as Eq. (89) to hold, since the null rays eventually do run into a singularity. This is assuming, of course, that the "standard" scenario depicted in Fig. (3) is in fact the correct one - a view which could well be wrong. In our $2 \mathrm{D}$ analysis, we found that ANEC was satisfied along these rays, and also along ingoing null rays.

We showed that our approximation of neglecting backreaction should fail only when the time of escape of a null ray from near the horizon is comparable to the evaporation time of the black hole. This implied that such a ray would have to start out extremely close to the horizon. Nevertheless, it would be useful to attempt a similar analysis to ours for evaporating black hole spacetimes with backreaction taken into account. The situation is complicated by our ignorance of the detailed form of the stress-tensor components in such cases.

Recent work of Kuo and Ford 44, 46] has shown that negative energy densities in flat spacetime are subject to large fluctuations. In these circumstances, one does not expect the semi-classical Einstein equations to be a good approximation. If the same is true of negative energy densities in curved spacetime, and we see no reason to believe otherwise, then the question arises as to whether such fluctuations could cause 
a gross failure of the semi-classical approximation at scales well above the Planck scale. Large stress-tensor fluctuations would presumably produce large fluctuations in spacetime geometry. Therefore, it is very important to determine whether such fluctuations might render suspect the semi-classical picture of the Hawking process, or the conclusions of the singularity theorems in the presence of negative energy. On the other hand it could turn out, for example in the Hawking process, that if the timescale of the stress-tensor fluctuations is very short compared to other relevant time scales, such as the evaporation time of the hole, then the standard analysis may still be valid. In the proofs of the singularity theorems, one assumes a fixed classical spacetime background which determines the causal structure. If the background geometry fluctuates, it is not clear that the usual global techniques employed in classical general relativity will still be useful [47]. These questions are currently under active investigation.

\section{Acknowledgements}

The authors would like to thank Arvind Borde, Eanna Flanagan, Matt Visser, Bob Wald, and Ulvi Yurtsever for helpful discussions. TAR would like to thank the members of the Tufts Institute of Cosmology for their kind hospitality and encouragement while this work was being done. This research was supported in part by NSF Grant No. PHY-9208805 (Tufts), by the U.S. Department of Energy (D.O.E.) under cooperative agreement \# DF-FC02-94ER40818 (MIT), and by a CCSU/AAUP Faculty Research Grant.

\section{References}

[1] S.W. Hawking and G.F.R. Ellis, The Large Scale Structure of Spacetime (Cambridge University Press, London, 1973), pp. 88-96.

[2] H. Epstein, V. Glaser, and A. Jaffe, Nuovo Cim. 36, 1016 (1965).

[3] H.B.G. Casimir, Proc. Kon. Ned. Akad. Wet. B51, 793 (1948).

[4] F.J. Tipler, Phys. Rev. D 17, 2521 (1978).

[5] C. Chicone and P. Ehrlich, Manuscr. Math. 31, 297 (1980).

[6] G.J. Galloway, Manuscripta Math. 35, 209 (1981).

[7] A. Borde, Class. Quantum Grav. 4, 343 (1987).

[8] T.A. Roman, Phys. Rev. D 33, 3526 (1986).

[9] T.A. Roman, Phys. Rev. D 37, 546 (1988).

[10] J. Friedman, K. Schleich, and D. Witt, Phys. Rev. Lett. 71, 1486 (1993). 
[11] Note that in a general spacetime, unlike the relation between the local null and weak energy conditions, ANEC does not simply follow by continuity from AWEC. An example of this is illustrated in Sec. (2) for the Unruh vacuum state.

[12] G. Klinkhammer, Phys. Rev. D 43, 2542 (1991).

[13] A. Folacci, Phys. Rev. D 46, 2726 (1992).

[14] R. Wald and U. Yurtsever, Phys. Rev D 44, 403 (1991).

[15] M. Visser, Phys. Lett. B349, 443 (1995).

[16] U. Yurtsever, Phys. Rev. D 51, 5797 (1995).

[17] U. Yurtsever, Phys. Rev. D 52, R564 (1995).

[18] M. Morris and K. Thorne, Am. J. Phys. 56, 395 (1988).

[19] M. Morris, K. Thorne, and U. Yurtsever, Phys. Rev. Lett. 61, 1446 (1988).

[20] L. Ford and T. Roman, Phys. Rev. D, 51, 4277 (1995).

[21] L.H. Ford, Proc. Roy. Soc. Lond. A 364, 227 (1978).

[22] L.H. Ford, Phys. Rev. D 43, 3972 (1991).

[23] This particular choice was initially made in Ref. 222] purely for mathematical convenience. Presumably, one could prove analogous inequalities with other choices of sampling functions.

[24] L.H. Ford and T.A. Roman, Phys. Rev. D 41, 3662 (1990).

[25] L.H. Ford and T.A. Roman, Phys. Rev. D 46, 1328 (1992).

[26] L.H. Ford and T.A. Roman, Quantum Field Theory Constrains Traversable Wormhole Geometries, manuscript in preparation.

[27] U. Yurtsever, Class. Quantum Grav. 7, L251 (1990).

[28] R. Penrose, Phys. Rev. Lett. 14, 57 (1965).

[29] S. W. Hawking, Commun. Math. Phys. 43, 199 (1975).

[30] A. Borde, "Topology Change in Classical General Relativity", Tufts Institute of Cosmology preprint TUTP 94-13, gr-qc 9406053.

[31] L.H. Ford and T.A. Roman, Phys. Rev. D 48, 776 (1993).

[32] W.G. Unruh, Phys. Rev. D 15, 365 (1977). 
[33] Note that this expression differs somewhat in form from Eq. (10). However, the latter is an inequality valid for all quantum states in 2D Minkowski spacetime, whereas the integral in Eq. (27) is evaluated for a restricted class of geodesics in a specific quantum state in Schwarzschild spacetime. Note also that the righthand-side of Eq. (27) is more negative than the right-hand-side of Eq. (10). This is presumably due to the integration over a half-complete geodesic in Eq. (27); even in Minkowski spacetime, there exist states for which Eq. (10) would not be true if the integration were taken only over a half-geodesic.

[34] P.C.W. Davies and W.G. Unruh, Proc. Roy. Soc. Lond. A 356, 259 (1977).

[35] R. M. Wald, General Relativity (Univ. of Chicago Press, Chicago, 1984).

[36] R. M. Wald, Quantum Field Theory in Curved Spacetime and Black Hole Thermodynamics (Univ. of Chicago Press, Chicago, 1994).

[37] U. Yurtsever, private communication.

[38] T. Elster, Phys. Lett. 94A, 205 (1983).

[39] B.P. Jensen, J.G. McLaughlin, and A.C. Ottewill, Phys. Rev. D 43, 4142 (1991).

[40] M. Visser, Lorentzian Wormholes - from Einstein to Hawking (American Institute of Physics Press, New York, 1995), Sec. 12.3.6, pp. 128-9.

[41] The form of Eq. (63) is motivated by the following considerations: If we imagine a sequence of observers shot outward with increasing values of $k$, then $T_{\mu \nu} u^{\mu} u^{\nu} \propto k^{2}$ and $\delta \tau \propto k^{-1}$, so $\int T_{\mu \nu} u^{\mu} u^{\nu} d \tau \propto\left(k^{2}\right) k^{-1} \propto(\delta \tau)^{-1}$. The factor of $M^{-2}$ is required in order for the dimensions to be correct. This form is also suggested by the form of the inequality found for orbiting null geodesics, Eq. (85).

[42] D. N. Page, Phys. Rev. D13, 198 (1976).

[43] A. Borde, private communication.

[44] See p. 56, footnote 2 of R. Penrose, Techniques of Differential Topology in Relativity (Society for Industrial and Applied Mathematics, Philadelphia, 1972), for an interesting example with timelike geodesics.

[45] C.-I Kuo and L.H. Ford, Phys. Rev. D 47, 4510 (1993).

[46] C.-I Kuo, Quantum Fluctuations and Semiclassical Gravity Theory, Ph.D. thesis, Tufts University (1994), unpublished.

[47] The authors are grateful to A. Borde for this remark. 


\section{Figure Captions}

- [1] Radial null geodesics in the spacetime of a black hole formed by gravitational collapse: an ingoing geodesic (a), an outgoing geodesic inside the horizon (b), and an outgoing geodesic outside of the horizon (c).

- [2] The spacetime of a black hole formed by gravitational collapse. The null ray which lies in the future horizon enters the collapsing body (shaded region), and then re-emerges into the vacuum spacetime at $V_{\min }$.

- [3] The spacetime of an evaporating black hole, including the effects of backreaction. 
This figure "fig1-1.png" is available in "png" format from: http://arxiv.org/ps/gr-qc/9506052v2 
This figure "fig1-2.png" is available in "png" format from: http://arxiv.org/ps/gr-qc/9506052v2 
This figure "fig1-3.png" is available in "png" format from: http://arxiv.org/ps/gr-qc/9506052v2 